\title{
Fluorescence Imaging Methods to Investigate Translation in Single Cells
}

\author{
Jeetayu Biswas, ${ }^{1,5}$ Yang Liu, ${ }^{2,5}$ Robert H. Singer, ${ }^{1,3,4}$ and Bin $\mathrm{Wu}^{2}$ \\ ${ }^{1}$ Department of Anatomy and Structural Biology, Albert Einstein College of Medicine, Bronx, New York 10461 \\ ${ }^{2}$ Department of Biophysics and Biophysical Chemistry, Center for Cell Dynamics, Department of Neuroscience, \\ Johns Hopkins School of Medicine, Baltimore, Maryland 21218 \\ ${ }^{3}$ Gruss Lipper Biophotonics Center, Albert Einstein College of Medicine, Bronx, New York 10461 \\ ${ }^{4}$ Howard Hughes Medical Institute, Janelia Research Campus, Ashburn, Virginia 20147 \\ Correspondence: bwu20@jhmi.edu
}

Translation is the fundamental biological process that converts the genetic information in messenger RNAs (mRNAs) into functional proteins. Translation regulation allows cells to control when, where, and how many proteins are synthesized. Much of what we know about translation comes from ensemble approaches that measure the average of many cells. The cellular and molecular heterogeneity in the regulation of translation remains largely elusive. Fluorescence microscopy allows interrogation of biological problems with single-molecule, single-cell sensitivity. In recent years, improved design of reagents and microscopy tools has led to improved spatial and temporal resolution of translation imaging. It is now possible to track global translation in specific subcellular compartments and follow the translation dynamics of single transcripts. Highlighted here is the recent progress in translation imaging with emphasis on in vivo translation dynamics. These tools will be invaluable to the study of translation regulation.

$T^{\text {: }}$ ranslation of messenger RNAs (mRNAs) into proteins is precisely controlled in space and time. During development, many mRNAs localize to specific subcellular regions. During Drosophila embryogenesis, for instance, over $70 \%$ of tested mRNAs are compartmentalized (Lecuyer et al. 2007). To maintain proteome homeostasis, cells respond to environmental cues by translating existing mRNAs without drastically altering transcription rates. For example, mRNAs encoding ribosomal proteins are highly enriched at the apical surface of fasted intestinal epithelium cells and feeding induces a global basal-to-apical shift of mRNAs and increased translation efficiency (Moor et al. 2017). In neurons, high-resolution fluorescence in situ hybridization (FISH) data show a tight correlation between the spatial distribution of mRNAs and their final protein products, suggesting a significant role of mRNAs in protein localization. Deep RNA sequencing (RNA-Seq) has identified more than 2500 mRNAs localized in neuronal dendrites and axons (Cajigas et al. 2012) and, when combined with proteomics, has

\footnotetext{
${ }^{5}$ These authors contributed equally to this work.

Editors: Michael B. Mathews, Nahum Sonenberg, and John W.B. Hershey

Additional Perspectives on Translation Mechanisms and Control available at www.cshperspectives.org

Copyright $\odot 2019$ Cold Spring Harbor Laboratory Press; all rights reserved; doi: 10.1101/cshperspect.a032722

Cite this article as Cold Spring Harb Perspect Biol 2019;11:a032722
} 
J. Biswas et al.

shown that neurite-targeted RNAs encode approximately half of the neurite-localized proteome (Zappulo et al. 2017). Local translation regulates many neuronal processes such as growth cone motility and synaptic plasticity (Holt and Schuman 2013). For instance, treatment with nerve growth factor (NGF) stimulates axonal elongation and pathfinding by trafficking and locally translating Par3 mRNAs in developing axons (Hengst et al. 2009). To understand these molecular processes and biological functions, it is necessary to track when and where specific mRNAs are translated and how newly synthesized proteins are distributed.

Much of our knowledge about the mechanisms underlying translation derives from standard and sophisticated biochemical and molecular biology methods. Various proteins and nucleic acids involved in translation have been purified and reconstituted for in vitro mechanistic studies (Chen et al. 2016). Recently, highthroughput assays such as ribosome profiling have revolutionized our understanding of translation by providing the global translation status of mRNAs (Brar and Weissman 2015; Ingolia et al. 2018). In addition, stable isotope labeling with amino acids in cell culture (SILAC) allows newly synthesized proteins to be quantified genome-wide by quantitative mass spectrometry (Schwanhausser et al. 2011). Although powerful and successful, these ensemble measurements detect overall changes in protein synthesis across millions of cells. Translational information regarding spatiotemporal kinetics, especially cellto-cell and molecule-to-molecule heterogeneity, have remained largely elusive because of the lack of molecular tools with sufficient resolution and sensitivity.

Fluorescence microscopy has emerged as an ideal tool to complement the aforementioned ensemble approaches. Instead of taking a snapshot of a biological event, imaging studies the translation process in real time with a temporal resolution of milliseconds to hours. The entire process of translation-initiation, elongation, pausing, and eventual termination-can be examined with imaging. Fluorescence imaging records the location of translation with high spatial resolution comparable to the size of a ribo- some (diameter $25-30 \mathrm{~nm}$ ). Furthermore, the distribution of newly synthesized protein can be followed in vivo, which offers significant insight into the molecular mechanism. Recent technological advances in fluorescence microscopy have made it possible to visualize single molecules. Fluorescently tagged ribosomes, mRNAs, and newly synthesized proteins have been individually labeled and tracked with single-molecule sensitivity.

In this review, we highlight recently developed microscopy techniques for translation imaging in single cells. These techniques are classified as either pulse-chase approaches or genetically encoded approaches (as summarized in Table 1). We compare these methods and provide a brief outlook regarding possible improvements and new insights that may arise from them.

\section{PULSE-CHASE-BASED APPROACHES}

To study the synthesis of protein products, one has to distinguish newly synthesized proteins from preexisting ones. A classical method for achieving this is through the pulse-chase approach, in which newly synthesized proteins are specifically tagged. With this approach, the localization of newly synthesized proteins and the distribution of translation-rich regions (hot spots) can be determined. We broadly divide these techniques into genetically encoded and nongenetically encoded groups (Fig. 1A,B).

\section{Nongenetically Encoded Approaches}

The nongenetic imaging approaches (Fig. 1A) allow visualization of the translation of endogenous mRNAs without any genetic modification. These approaches are extensively used to study the spatial distribution of the translation sites and newly produced proteins.

Classically, isotopically labeled amino acids have been powerful tools to study global protein synthesis. The highly specific vibrational energy of a carbon-deuterium bond is amenable to measurement by stimulated Raman scattering (SRS) microscopy (Wei et al. 2013) and pulsed metabolic incorporation of deuterated amino 
Imaging Translation in Single Cells

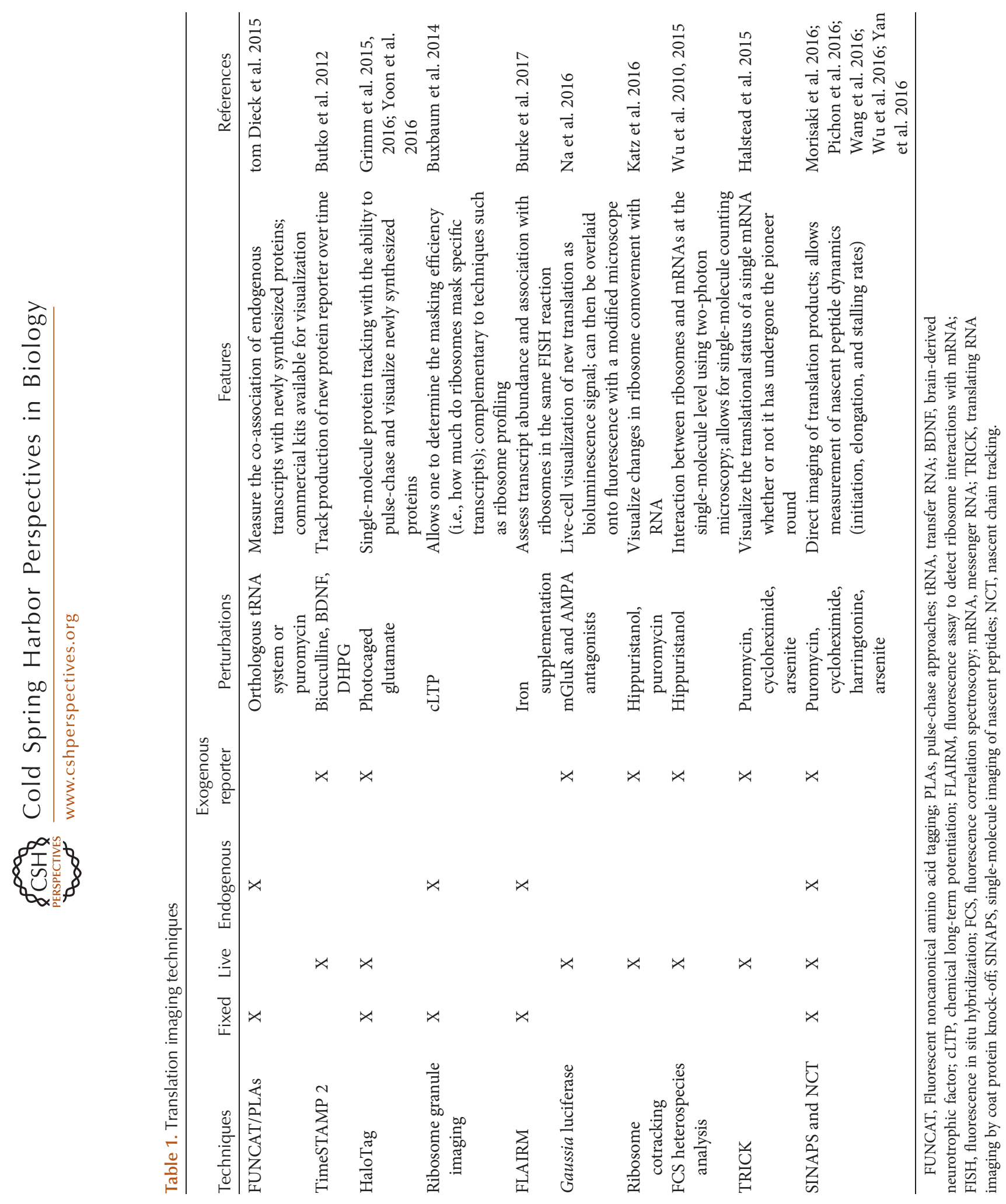


J. Biswas et al.
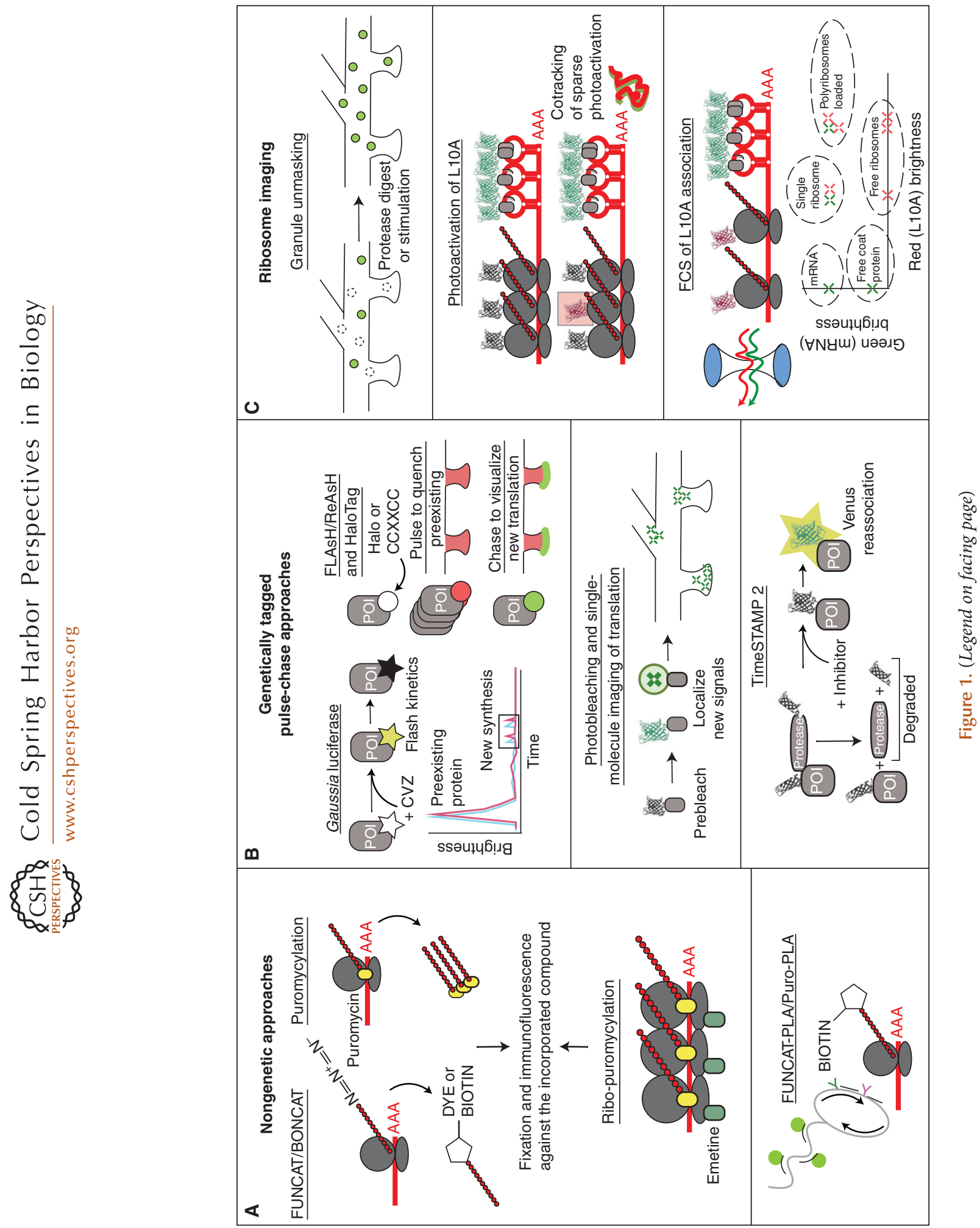
acid allows nascent protein synthesis to be visualized in live cells. Newly synthesized proteins are highly concentrated in nucleoli, the sites of ribosome biogenesis. The production of ribosomes is a major task in rapidly growing cells and the majority of the newly synthesized nucleolar-localized proteins are presumably ribosomal proteins that are trafficked from the cytoplasm, in agreement with previous observations of fast proteomic turnover in nucleoli (Beatty et al. 2006; Boisvert et al. 2012). The technique has also been generalized to other vibrational tags such as alkynes, allowing protein, RNA, and DNA synthesis to be tracked simultaneously in live cells (Wei et al. 2014).

In fluorescent noncanonical amino acid tagging (FUNCAT; Fig. 1A), azidohomoalanine (AHA), a methionine analog, is incorporated into all newly synthesized proteins in methionine-starved cells. Subsequent fluorescent labeling by a copper-catalyzed click chemical reaction or immunofluorescence allows visualization of the newly synthesized proteins (Dieterich et al. 2010). Puromycylation uses a similar conceptual approach (Fig. 1A). Instead of using methionine-specific labeling, nascent protein synthesis is truncated by low-dose treatment with puromycin, which enters the ribosomal A site and forms a peptide bond with the nascent chain (Starck et al. 2004). The incorporation of puromycin into the newly synthesized nascent proteins is then visualized with fluorescent antipuromycin antibodies (Schmidt et al. 2009). Alternatively, an alkyne analog of puromycin, $O$-propargyl-puromycin (OP-puro) can form covalent conjugates with nascent peptides, which can be visualized by an azide-labeled fluorophore via click chemistry (Liu et al. 2012).

FUNCAT has been used to observe local translation in different neuronal compartments. Upon stimulation of brain-derived neurotrophic factor, local production of proteins, such as CamKII $\alpha$, increases in postsynaptic dendrites (Dieterich et al. 2010). By combining FUNCAT with a microfluidic device that allows axons to be physically isolated from other neuronal compartments, stimulation-dependent local translation was observed in both axons and dendrites

Figure 1. Techniques to visualize translation at the single-cell and single-molecule level. $(A)$ Both puromycylation and noncanonical amino acid tagging (NCAT) techniques incorporate a small molecule ligand into the protein of interest. For puromycylation, this is a carboxy-terminal puromycin (yellow oval), and for NCAT techniques, this is an amino-terminal azide group that is incorporated into the nascent chain and then can be conjugated to a dye or biotin moiety. To visualize the location of translating polysomes, emetine can be added before fixation (green oval) to allow for puromycin incorporation but prevent ribosome dissociation from the transcript. Conversely, one can use two oligonucleotide-conjugated antibodies to create a bridge for rolling circle amplification and the coincident locations can then be identified by fluorescent dye (green circles)-labeled fluorescence in situ hybridization (FISH) probes. (B) Small molecule dyes or substrates can be used to quench the previously synthesized proteins of interest. For HaloTag and FLAsH ReAsH, the second optically separated dye (green circle) identifies the newly translated proteins. For Gaussia luciferase, the substrate (yellow star) is rapidly inactivated (black star) leading to previously synthesized proteins being irreversibly in the dark state and newly synthesized proteins being observed as small flashes of luminescence (small peaks on graph). One can also use continuous photobleaching of fluorescent proteins to isolate only newly synthesized signal. By repeatedly localizing the proteins of interest, it is possible to create maps depicting the translation hot spots (be they at the synapse, neck of the spine, or at dendritic branch points). A similar dark-to-bright approach can be used when a protease site is placed between the two halves of Venus. Normally, continuous protease degradation prevents the two halves of Venus from associating. Upon addition of an inhibitor, one can visualize newly formed proteins by fluorescence rescue (yellow star). (C) Direct and indirect imaging of ribosomes. FISH probes have limited permeability because of the density of granule components in neuronal dendrites, and after chemical longterm potentiation (LTP) or protease digestion granule unmasking exposes an increased number of messenger RNAs (mRNAs) (green circles). Sparse photoactivation of tagged ribosomes can allow for multicolor cotracking of ribosomes associated with single mRNA molecules. Fluorescence correlation spectroscopy (FCS) allows accurate counting of individual molecules as they traverse the femtoliter excitation volume. Heterospecies partition analysis allows one to determine the percentages of transcripts that are associated with ribosomes as well as count the number of ribosomes associated with each transcript. 
J. Biswas et al.

(Kos et al. 2016). Others have applied FUNCAT to measure translation in brain slices, showing that it occurs in both pre- and postsynaptic compartments. Using this approach, it was found that presynaptic, cap-dependent protein synthesis is required for long-term but not short-term plasticity of a specific population of GABA-releasing axons (Younts et al. 2016). Injection of OP-puro into mice allows protein synthesis to be tracked in whole organisms (Liu et al. 2012). In the mouse intestine, OP-puro strongly stained the cells in the crypts and at the base of the villi and was strongly incorporated into striated muscle fibers.

To observe translating ribosomes, the translation elongation inhibitor emetine was added in combination with puromycin to prevent release of the nascent chain (David et al. 2012; Graber et al. 2013). Using this combined inhibition, dubbed "ribopuromycylation" (Fig. 1A), David et al. (2012) observed a nuclear translation signal. By studying the process in intact cells and avoiding possible contamination from endoplasmic reticulum (ER)-associated ribosomes, the investigators proposed the existence of nuclear translation, a controversial observation needing further independent validation.

Both FUNCAT and puromycylation measure the bulk translation status of the entire proteome rather than the production of specific proteins. Recently, a proximity ligation assay was combined with pulse-chase approaches (FUNCAT-PLA or Puro-PLA) to overcome this disadvantage (tom Dieck et al. 2015). In this work, cells are fixed and incubated with two different primary antibodies. The first antibody detects a protein of interest and the second detects biotin or puromycin, which is followed by the addition of two secondary antibodies conjugated with corresponding DNA oligonucleotides. Only when both secondary antibodies are bound to the same molecule (in close proximity, $10-20 \mathrm{~nm}$ ), is an enzymatic reaction named rolling circle amplification (RCA) initiated to produce thousands of identical copies of DNA sequences (Fig. 1A, Table 1). Through this strategy, newly synthesized proteins are visualized by fluorescently labeled probes binding to RCA products.
The synthesis of new proteins is required for stabilizing synapses during long-term potentiation and long-term depression. FUNCAT-PLA has been used to visualize local translation of proteins in the soma as well as the neurites. The presynaptically localized protein Bassoon was thought to be translated in the soma and posttranslationally localized to presynapses. FUNCAT-PLA showed that newly synthesized Bassoon was locally made in the presynaptic bouton (tom Dieck et al. 2015). The ability to pulse-chase at different time points has allowed visualization of proteins that are locally translated in the soma and then subsequently localized to the neurites, such as the protein Barentsz, which is a component of the RNA localization machinery (Zappulo et al. 2017).

\section{Genetically Tagged Pulse-Chase}

Genetic modification allows incorporation of fluorescent tags into target genes, which gives rise to highly specific fluorescence signals (Fig. 1B). Recently, genetically encoded protein motifs have been designed to bind different ligands. Researchers use this property to distinguish newly synthesized proteins from preexisting ones by pulse-chase labeling with different fluorophores. An example is the tetracysteine motif CCXXCC (where X represents any amino acid), which binds biarsenical dyes with picomolar affinity and converts them from a nonfluorescent to a fluorescent state (Griffin et al. 1998). In a FlAsH-ReAsH system, green (FlAsH) and red $(\mathrm{ReAsH})$ dyes were used in a pulse-chase fashion to determine that $\beta$-actin translation sites are more frequently located at cell contacts (Fig. 1B) (Rodriguez et al. 2006). The tiny tetracysteine tag causes minimal perturbation to the function of proteins of interest. However, the arsenical dyes suffer from low brightness and are toxic to cells and can only be used for short-term experiments with relative low resolution. More recently, covalent labeling techniques such as HaloTag (as well as SNAP-tag, CLIP, and TMP) (Gautier et al. 2008; Los et al. 2008; Gallagher et al. 2009) allows irreversible conjugation of bright organic fluorophores such as the Janelia Fluors (JF dyes) (Grimm et al. 
Imaging Translation in Single Cells

2015, 2016). These dyes are $10 \times$ brighter than green fluorescent protein (GFP) and allow single-molecule detection in live cells. Based on pulse-chase labeling of HaloTag ligands, translation of newly synthesized proteins has been visualized during synaptic stimulation (Yoon et al. 2016). Caged glutamate molecules (4-methoxy-7-nitroindolinyl-moiety) were photolyzed with a $405 \mathrm{~nm}$ laser to stimulate single dendritic spines, which induces rapid localization of endogenous $\beta$-actin mRNAs. After preexisting $\beta$ actin-HaloTag reporter proteins were covalently labeled to saturation with the far-red dye JF-646, a second HaloTag-ligand (the near-red dye JF549) was used to detect newly synthesized proteins (Fig. 1B, Table 1) (Grimm et al. 2015). The investigators showed that synaptic activity induces local translation of $\beta$-actin, which plays a critical role in stabilizing the growth of dendritic spines.

In addition to traditional pulse-chase approaches that use small molecules as ligands, fluorescent proteins have been used to track newly synthesized proteins. An early approach to visualize translation observed changes in fluorescence intensity over time (Aakalu et al. 2001), which has limited capability to distinguish old proteins from newly synthesized ones. To overcome this limitation, different strategies such as photoconversion or photobleaching have been used. These light-up tags include photoconvertible proteins, fluorescent timers, and TimeSTAMP (discussed briefly here as well as in many other reviews [Wu et al. 2011; Zhou and Lin 2013]). To visualize translation of the mRNA encoding the peptide neurotransmitter Sensorin, its $5^{\prime}$ and $3^{\prime}$ untranslated regions (UTRs) were fused to the coding region of Dendra2 (a photoconvertible protein). Conversion of the preexisting proteins (from green to red) with a pulse of ultraviolet (UV) illumination allowed newly synthesized proteins to be visualized in Aplysia motor neurons. Translation of this reporter was found to be highly regulated, requiring calcium in the postsynaptic motor neurons, and to occur during long-term facilitation but not during long-term depression (Wang et al. 2009). More recently, the rapid maturation of a yellow fluorescent protein mutant (named Venus) has been exploited to visualize translation at the single-molecule level. By photobleaching the preexisting Venus fusion protein, it has been possible to localize and observe short bursts of fluorescence from newly synthesized reporter proteins (Yu et al. 2006). By mapping spot localizations over time, translation maps, which mark sites of new protein synthesis, have been made for Arc (activity-related cytoskeleton-associated protein), fragile $\mathrm{X}$ mental retardation protein (FMRP), postsynaptic density protein 95 (PSD-95) and $\beta$-actin (Fig. 1B). The clustering of translation events was found, either near granules for FMRP and Arc (Tatavarty et al. 2012), synapses for PSD-95 (Ifrim et al. 2015), or dendritic branch points for $\beta$ actin (Wong et al. 2017). When using this technique, one must carefully control the cyclical "blinking" of fluorescent proteins.

In addition to reducing preexisting protein signals by photobleaching (or photoconversion), small-molecule-controlled degradation has been adopted as another strategy to remove previously translated proteins. In TimeSTAMP, the nonstructural protease 3 (NS3) of hepatitis C virus is fused to a protein of interest to degrade it by default. Addition of a protease inhibitor stabilizes the target protein and makes it detectable (Lin et al. 2008). TimeSTAMP 2 uses a split fluorescent protein Venus and irreversible bimolecular fluorescence complementation for live cell translation imaging (Butko et al. 2012). With the addition of the protease inhibitor, the two halves of Venus have enough time to come together and the appearance of the fluorescent signal can be used to track newly translated proteins (Fig. 1B, Table 1).

\section{GENETICALLY ENCODED REPORTERS TO VISUALIZE RNAs BEFORE, AFTER, AND DURING TRANSLATION}

Fluorescence techniques have been developed to visualize ribosomes and mRNAs at the singlemolecule level. FISH is able to detect single endogenous mRNAs or ribosomes in a fixed sample using multiple fluorescently labeled oligonucleotides directed against target transcripts or ribosomal RNAs (rRNAs) (Buxbaum et al. 
J. Biswas et al.

2014). To image ribosomes or RNA in live cells, genetically encoded fluorescent tags are used. To visualize single mRNAs, MS2-binding sites (MBS) derived from bacteriophage MS2, are inserted as an array of 24 repeats into the $3^{\prime}$ UTR of the target gene (Bertrand et al. 1998). Multiple GFP-fused MS2 coat proteins (MCPs) bind to MBS and allow single-mRNA detection. Recently, modified MS2 stem-loops have been developed to follow unstable mRNAs from transcription to degradation (Tutucci et al. 2017). In addition to MS2, other RNA-binding proteins (Daigle and Ellenberg 2007; Chao et al. 2008) are also used for single-molecule tracking. In the next section, we discuss the application of these fluorescence labeling technologies in translation imaging.

\section{IMAGING RIBOSOMES AS A READOUT FOR TRANSLATION}

RNAs are complexed with proteins and form cellular particles, ribonucleoproteins (RNPs), and neuronal RNA granules. Granules contain ribosomal subunits but are devoid of initiation factors and tRNAs necessary for active translation (Krichevsky and Kosik 2001). By sequestering RNAs and the required translational machinery into a repressed transporting granule, translational control is achieved both in time and space by relieving the repression (see also Ivanov et al. 2018). It is still unclear how ribosomes interact with the RNA inside the granule. Possibly, the granule represents the accumulation of previously initiated but stalled ribosomes (Graber et al. 2013). Masking of RNA transcripts has long been thought to control translation (Anderson and Kedersha 2006; Kiebler and Bassell 2006; Ivanov et al. 2018). Chemical stimulation rapidly and transiently increases the number of $\beta$-actin mRNAs in the neuronal dendrite observable by FISH (Fig. 1C, Table 1). FISH against rRNA showed that $\beta$-actin mRNA was indeed present in a masked state and that stimulation lowered the rRNA density around the mRNA (granule unmasking) (Buxbaum et al. 2014).

Techniques that visualize the interaction of specific mRNAs with ribosomes have been de- veloped. Fluorescence assay to detect ribosome interactions with mRNA (FLAIRM) uses FISH to detect ribosomes in close proximity with the target mRNAs (Burke et al. 2017). A linker oligonucleotide bridges FISH probes targeted to ribosomes and to mRNAs. Signal amplification is achieved by the hybridization chain reaction only when the ribosomes and mRNAs are close to each other. The investigators used this technique to simultaneously track changes in the abundance of the endogenous ferritin heavy chain transcript as well as its ribosome association. They found that, on addition of exogenous iron, transcript abundance increased only modestly, but the mRNA-ribosome interaction increased significantly, indicating enhanced translation.

In live cells, the association between ribosome and mRNA can be inferred if they move together. Abundant ribosomal signals preclude tracking of a single ribosome. One approach is to label ribosomal protein with a photoactivatable fluorescent tag. After sparse photoactivation, single ribosomes can be localized with $\sim 20 \mathrm{~nm}$ precision (Fig. 1C) and the coordinate movements of photoactivated ribosomes and mRNAs tracked. This allowed Katz et al. (2016) to infer when and where translation occurred. The slower, corralled movements of the mRNA-ribosome complex correlate with more active translation of $\beta$-actin mRNA around the focal adhesion complex. The association between ribosomes and mRNAs has also been studied with fluorescence fluctuation spectroscopy (FFS). A focused two-photon laser captures bursts of fluorescence when single molecules diffuse in and out of the femtoliter excitation volume. When two interacting molecules pass through the observation volume, their fluorescence signals are detected simultaneously on different detectors (Fig. 1C, Table 1). Statistical analysis is applied to extract interaction information between molecules. By labeling $\beta$-actin mRNA in one color and ribosomes in another, and performing subsequent heterospecies partition analysis ( $\mathrm{Wu}$ et al. 2010), the average numbers of ribosomes associated with mRNAs can be measured ( $\mathrm{Wu}$ et al. 2015). More ribosomes associate with $\beta$-actin mRNA at the periphery of the cell than 
A
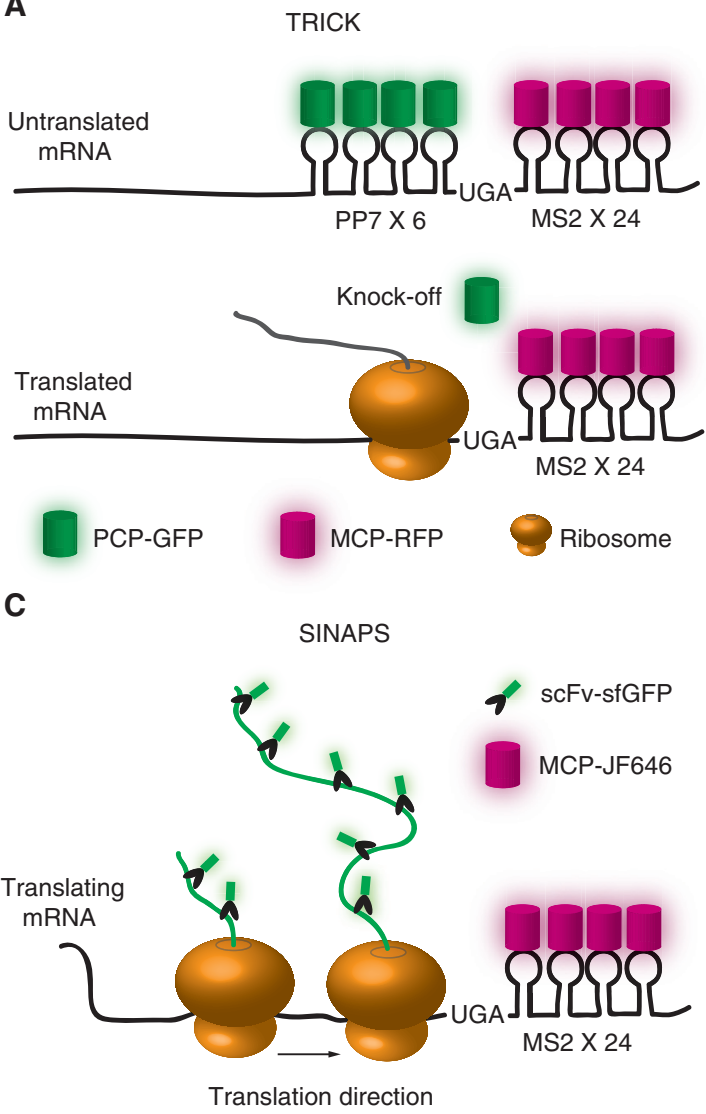

TRICK

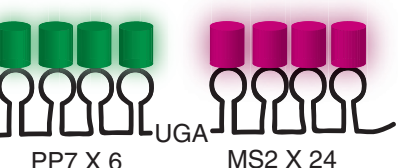

B
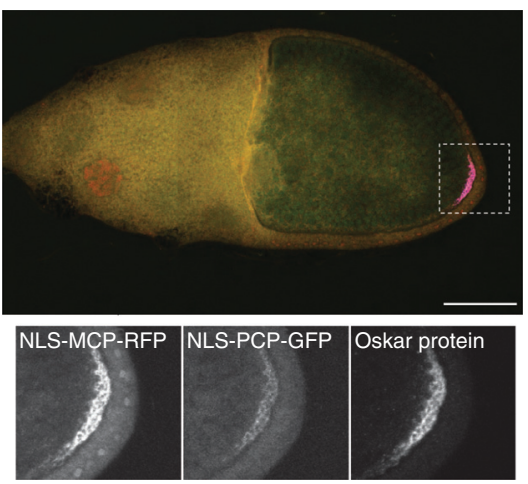

D

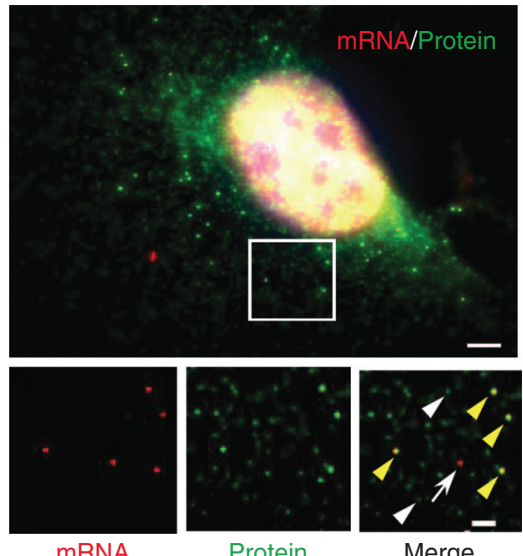

Protein

Merge

Figure 2. Real-time translation imaging using fluorescent reporter systems. (A) Working principle of translating RNA imaging by coat protein knock-off (TRICK). Messenger RNAs (mRNAs) are fused with PP7 bacteriophage stem-loops in the coding region and MS2 stem-loops in the $3^{\prime}$ untranslated region (UTR), to which PP7 coat proteins (PCPs)-GFP (green) and MS2 coat proteins (MCPs)-RFP (red) are bound, respectively. During the pioneer round of translation, ribosomes displace the PCP-GFP and, as a result, a subpopulation of mRNAs lose their green fluorescent signal and retain only their red fluorescent signal, indicating that they had been translated at least once. (B) TRICK imaging of Oskar protein expression at the pole of stage 10 Drosophila oocyte. Scale bar, $50 \mu \mathrm{m}$. (Panel $B$ from Halstead et al. 2015; adapted, with permission, from HHS Public Access and Science ( $)$ 2015.) (C) Working principle of single-molecule imaging of nascent peptides (SINAPS). Genes of interest are fused with multiple peptidyl epitopes recognized by specific GFP-tagged antibodies. When the nascent peptides emerge from ribosomes, coexpressed fluorescent antibodies (green) spontaneously bind to these tandem epitope repeats, yielding a bright fluorescent spot colocalizing with the MS2/PP7 tagged mRNAs (red). (D) SINAPS imaging of translation heterogeneity in living U2OS cells. Translating mRNAs appear in both green (scFv-GFP) and red (MCP-RFP) channels, whereas nontranslating mRNAs appear only in red because of the absence of antibodies. Scale bars, $5 \mu \mathrm{m}$ (top); $2 \mu \mathrm{m}$ (lower right). (Panel D from Wu et al. (2016); adapted, with permission, from HHS Public Access and Science @ 2016.$)$

in the perinuclear region. This is anticorrelated with the mRNA binding to a well-known translational repressor, the RNA-binding protein, zipcode-binding protein $\mathrm{ZBP} 1$, showing how a trans-acting factor can regulate the subcellular location of translation.

\section{DYNAMIC APPROACHES TO MEASURE TRANSLATION KINETICS IN LIVE CELLS}

Recent technological advances make it possible to measure translation dynamics of single mRNAs in live cells. Halstead et al. (2015) devel- 
J. Biswas et al.

oped a biosensor to directly visualize the first round of translation based on multicolor RNA labeling. The first round of translation, which could happen on both the cap-binding complex (CBC)- and eIF4E-bound mRNAs (Rufener and Mühlemann 2013), is critical for short-lived and responsive proteins (such as those of the cellular immediate early genes) and has been ascribed a role in mRNA quality control through nonsensemediated mRNA decay (reviewed by Karousis and Mühlemann 2018). Specific mRNAs were tagged with bacteriophage PP7 stem-loops in the coding region and MS2 stem-loops in the $3^{\prime}$ UTR and bound by spectrally distinct capsid proteins (green and red, respectively). Nuclear mRNAs and untranslated cytoplasmic mRNAs have both green and red colors. During the first round of translation, ribosomes displace the GFP-fused PP7 capsid protein (PCP-GFP). As a result, $\mathrm{mRNAs}$ that have been translated at least once will carry a single color (red, MCP-red fluorescent protein $[R F P])$. Interestingly, spatial profiling of these transcripts suggested many mRNAs underwent initial translation away from the nucleus. In contrast, when translation inhibitors (cycloheximide or puromycin) were preincubated with the cells, over $90 \%$ of cytoplasmic mRNAs retained both capsid proteins and were fluorescent in both colors, indicating they remained untranslated. The translation of mRNAs in special cell compartments such as stress granules can be seen using this biosensor, a technique termed translating RNA imaging by coat protein knock-off (Halstead et al. 2015) (TRICK; Fig. $2 \mathrm{~A}$ ). When the TRICK biosensor was constituted with a stress-responsive $5^{\prime}$ terminal oligopyrimidine (TOP) motif and expressed in living cells, greater than $10 \%$ of TOP mRNA transcripts were found strongly associated with processing bodies (P-bodies) and translationally repressed on arsenite treatment. More surprisingly, approximately $80 \%$ of these associated transcripts remained untranslated even after arsenite relief, suggesting a granule-based mechanism for translation regulation. The investigators further engineered an oskar-TRICK reporter to monitor the spatiotemporal translation regulation of Oskar protein during Drosophila development. It appeared that the initial round of translation only occurred lo- cally after mRNAs trafficked to the posterior pole area of a stage 10 oocyte (Fig. 2B), consistent with the detection of initial Oskar proteins. This method provides a general framework to visualize the spatial distribution and the kinetics of regulatory processes that are related with the first round of translation.

\section{Dynamic Translation Imaging Using \\ Bioluminescent and Fluorescent Reporter Systems}

Continuous monitoring of translation is necessary to follow protein production past the pioneer round. One approach to extend the observation time of translation used the ultrabright bioluminescence flashes generated by Gaussia luciferase (Fig. 1B, Table 1). When Gaussia luciferase oxidizes the small molecule substrate coelenterazine (CVZ), the luciferase is rapidly inactivated, thus attenuating the bright emission. Upon addition of excess CVZ, a bright flash of luminescence is generated by previously synthesized luciferase, which is then quickly inactivated. Subsequently, small quantal bursts of luminescence are observed, attributed to production of new proteins. $\mathrm{Na}$ et al. (2016) used this technique to study the translation of the immediate early protein Arc. Previous literature suggested that Arc transcription and translation are induced by neuronal stimulation. In agreement, the investigators found that new Arc synthesis was enhanced by glutamate stimulation and new translation often occurred repeatedly at the same sites, lending support to the idea of translation hot spots. Because these bursts of Arc translation were observed immediately after glutamate stimulation, the investigators concluded that translation of Arc is caused by previously stalled ribosomes that were poised and waiting for stimulation.

An alternative approach is to directly observe the synthesis of nascent proteins with multivalent fluorescent tags. This can be achieved either by immunofluorescence (IF), where multiple secondary antibodies recognize a primary antibody, or by introducing multiple epitopes to the amino terminus of a protein. One can confidently identify translation sites by colocalizing the IF and FISH signals. Chang et al. 2006 used FISH-IF 
as well as live-cell imaging to conclude that $30 \%$ of bright peripherin RNPs were sites of de novo peripherin synthesis before the protein was incorporated into intermediate filaments. Newer technologies have used the same conceptual approach to observe protein synthesis at the singlemolecule level.

In 2016, several laboratories simultaneously reported a fluorescence-based imaging technique to visualize translation dynamics of mRNAs in living cells, termed as either singlemolecule imaging of nascent peptides (SINAPS) or nascent chain tracking (NCT) (Morisaki et al. 2016; Pichon et al. 2016; Wang et al. 2016; Wu et al. 2016; Yan et al. 2016). Genes of interests are fused with multiple peptidyl epitopes recognized by specific antibodies. When fluorescently labeled antibodies and epitope-tagged genes are coexpressed in living cells, the antibodies spontaneously bind to the nascent peptides emerging from ribosomes. Multiple ribosomes on a single mRNA lead to bright translation sites. In SINAPS, three laboratories used an epitope that is targeted by a single-chain variable fragment $(\mathrm{scFv})$ fused with superfolder GFP ( $\mathrm{scFv}$ GCN4-sfGFP), which was previously termed as SUperNova or SunTag (Tanenbaum et al. 2014). This scFv recognizes the GCN4 epitope so as newly synthesized peptides exit the ribosome, multiple scFv-GCN4-sfGFP bind to the epitopes, yielding a bright fluorescent spot colocalizing with the MS2/PP7-tagged mRNAs (Fig. 2C, D). In NCT, Morisaki et al. constructed multimerized FLAG or HA peptides and imaged them with fluorescently labeled anti-FLAG or anti-HA Fab antibody fragments loaded into the cells (Viswanathan et al. 2015; Morisaki et al. 2016). This strategy allowed the investigators to perform multicolor imaging of translation.

To observe signals exclusively from nascent peptides, strategies have been devised to remove undesirable background signals from mature proteins. By adding an auxin-induced degron (AID) to the carboxyl terminus of the translation reporter, treatment with auxin causes the AID-containing mature protein to be rapidly degraded by the proteasome (Wu et al. 2016). Other researchers directly imaged the translation of a protein (ornithine decarboxylase) with a short lifetime (Wang et al. 2016). Both strategies achieved low backgrounds in the cytoplasm and reduced the sequestration of fluorescent antibodies to mature proteins. A third strategy immobilized PP7-tagged mRNAs onto the cell membrane through a CAAX palmitoylation motif. Because mature peptides diffused away from the cell membrane, the investigators also successfully imaged nascent peptide synthesis without any additional degradation strategies (Yan et al. 2016).

Compared to RNA-Seq and proteomic studies that assess an ensemble of RNAs and proteins, these fluorescence reporters display unique advantages. First, because of the singlemolecule sensitivity and resolution, individual mRNAs can be tracked for tens of minutes for the study of the spatial distribution of local translation in polarized cell types such as neurons. Wu et al. (2016) observed that the fraction of translating mRNAs is higher in proximal than distal dendrites. Second, fluorescence reporters provide unprecedented temporal resolution for tracking translation $(\sim 10-30 \mathrm{msec})$. The initiation and elongation rates of translation can be measured based on the fluctuation of fluorescence intensity. For example, these experiments measured ribosome elongation speed at around five amino acids per second and initiation rates in the range of once every 1-4 minutes. Third, translation can be recorded with single-molecule specificity. In many biological processes, it is only a subset of mRNAs that are translationally active at a given time, commonly resulting in a diminished correlation between mRNA and protein levels. In contrast, active and inactive mRNAs are distinguishable using these translation reporters. Therefore, these techniques are well suited for the study of events where translation of only one or a few mRNAs leads to a consequential biological phenotype.

In addition to the spatiotemporal resolution and molecular specificity, fluorescent reporters are especially powerful for studying translation mechanisms. One can investigate the mechanistic roles in translational control of different molecular elements (e.g., UTRs, cis elements) at the single-molecule level. For example, $\mathrm{Wu}$ et al. (2016) incorporated the $\beta$-actin $3^{\prime}$ UTR into a 
J. Biswas et al.

translation reporter and investigated localized translation of reporter mRNAs in neuronal dendrites. Yan et al. (2016) examined alternatively spliced forms of the $5^{\prime}$ UTR in Emil gene transcripts and found that for mRNAs with a long $5^{\prime} \mathrm{UTR}$, only $2 \%$ were translating and the rest were repressed. To understand how cis elements influence translation, Wang et al. (2016) engineered the stress-responsive cis element ATF4 to their reporter system. After DTT and arsenite treatment of the cell samples, they observed distinct translation dynamics of the mRNAs. Furthermore, two groups evaluated translation rates after adding regulatory sequences such as ERtargeting sequences and ribosome stalling sequences to the reporter systems (Wang et al. 2016).

Although the aforementioned reporter plasmids were transiently or stably transfected into cells, later work investigated translation of endogenous genes by inserting a SunTag-based translation reporter into a specific gene locus (Pichon et al. 2016). This capability will significantly expand the application of this technique to advance understanding of the role of translation in physiologically relevant events such as neurodegenerative diseases.

\section{CONCLUDING REMARKS/FUTURE DIRECTIONS}

Rapid technological advances in translation imaging have occurred because of a revolution in fluorescent reagents and microscopy. By tagging proteins of interest, researchers can track translation products and machineries. Through pulse-chase experiments, one can determine when and where new proteins are synthesized and how they are distributed. Multicolor RNA labeling allows visualization of the pioneering round of translation. Direct labeling of nascent multivalent peptides provides translation dynamics of single mRNAs in live cells. These powerful tools offer exciting opportunities to address questions about the translation dynamics and regulation. For example, how many times is an mRNA translated before it is degraded? The cytoplasmic fate of mRNA is closely related to its history in the nucleus, and researchers can now determine how different promoters, different splicing conditions, or covalent RNA modifications influence translation at the single-molecule level.

Scientists are continuously pushing the boundary of translation imaging. There are a few directions that require further developments. First, translation of single transcripts needs to be imaged continuously from their birth to death (Tutucci et al. 2018). A key parameter in biology is the number of proteins produced from a single mRNA. Various models and indirect measurements have given an estimation of this parameter. Single-molecule translation assays will provide definitive insight. Second, a quantitative model is necessary to integrate various aspects of an experiment. Totally asymmetric exclusion process (TASEP) has been used to model translation since the 1960s (Zia et al. 2011). It has been used to account for the size of ribosomes, rare codons, circulation of mRNA, finite resources of ribosomes, and tRNAs. Combining TASEP modeling with modern single-molecule translation assays would yield biological insights into the translation mechanism in vivo. Third, a method is needed to image the translation of endogenous transcripts. It has been observed that the cytoplasmic fate of an mRNA is intimately related to its nuclear history (Trcek et al. 2011). Translation may also depend on how the mRNA is produced, for example, transcribed from an endogenous promoter, spliced, edited, capped, and polyadenylated (Zid and O'Shea 2014). Ultimately, the goal is to image translation in live animals to study how endogenous mRNAs are regulated, for instance, during behavior. Local translation has been suggested to play an important role in neuronal plasticity, memory, and learning. It would be ideal to show the synthesis of certain key plasticityrelated proteins after the animal is subjected to cognitive challenges. Further development in reagents and microscopy tools will provide increasingly powerful new approaches to these questions.

\section{ACKNOWLEDGMENTS}

This publication is supported by National Institutes of Health (NIH) grants NS 83085 and GM 
57071 to R.H.S. J.B. is supported by NIH Medical Scientist Training Grant T32-GM007288 and Predoctoral Fellowship F30-CA214009. B.W. is supported by the Pew Biomedical Scholar program from Pew Charitable Trust. The authors also thank Dr. Carolina Eliscovich for discussion and suggestions.

\section{REFERENCES}

${ }^{*}$ Reference is also in this collection.

Aakalu G, Smith WB, Nguyen N, Jiang C, Schuman EM. 2001. Dynamic visualization of local protein synthesis in hippocampal neurons. Neuron 30: 489-502.

Anderson P, Kedersha N. 2006. RNA granules. J Cell Biol 172: 803-808.

Beatty KE, Liu JC, Xie F, Dieterich DC, Schuman EM, Wang Q, Tirrell DA. 2006. Fluorescence visualization of newly synthesized proteins in mammalian cells. Angew Chem Int Ed Engl 45: 7364-7367.

Bertrand E, Chartrand P, Schaefer M, Shenoy SM, Singer RH, Long RM. 1998. Localization of ASH1 mRNA particles in living yeast. Mol Cell 2: 437-445.

Boisvert FM, Ahmad Y, Gierlinski M, Charriere F, Lamont D, Scott M, Barton G, Lamond AI. 2012. A quantitative spatial proteomics analysis of proteome turnover in human cells. Mol Cell Proteomics 11: M111.011429.

Brar GA, Weissman JS. 2015. Ribosome profiling reveals the what, when, where and how of protein synthesis. Nat Rev Mol Cell Biol 16: 651-664.

Burke KS, Antilla KA, Tirrell DA. 2017. A fluorescence in situ hybridization method to quantify mRNA translation by visualizing ribosome-mRNA interactions in single cells. ACS Cent Sci 3: 425-433.

Butko MT, Yang J, Geng Y, Kim HJ, Jeon NL, Shu X, Mackey MR, Ellisman MH, Tsien RY, Lin MZ. 2012. Fluorescent and photo-oxidizing TimeSTAMP tags track protein fates in light and electron microscopy. Nat Neurosci 15: 17421751.

Buxbaum AR, Wu B, Singer RH. 2014. Single $\beta$-actin mRNA detection in neurons reveals a mechanism for regulating its translatability. Science 343: 419-422.

Cajigas IJ, Tushev G, Will TJ, tom Dieck S, Fuerst N, Schuman EM. 2012. The local transcriptome in the synaptic neuropil revealed by deep sequencing and high-resolution imaging. Neuron 74: 453-466.

Chang L, Shav-Tal Y, Trcek T, Singer RH, Goldman RD. 2006. Assembling an intermediate filament network by dynamic cotranslation. J Cell Biol 172: 747-758.

Chao JA, Patskovsky Y, Almo SC, Singer RH. 2008. Structural basis for the coevolution of a viral RNA-protein complex. Nat Struct Mol Biol 15: 103-105.

Chen J, Choi J, O'Leary SE, Prabhakar A, Petrov A, Grosely R, Puglisi EV, Puglisi JD. 2016. The molecular choreography of protein synthesis: Translational control, regulation, and pathways. Q Rev Biophys 49: e11.

Daigle N, Ellenberg J. 2007. $\lambda$ N-GFP: An RNA reporter system for live-cell imaging. Nat Methods 4: 633-636.
David A, Dolan BP, Hickman HD, Knowlton JJ, Clavarino G, Pierre P, Bennink JR, Yewdell JW. 2012. Nuclear translation visualized by ribosome-bound nascent chain puromycylation. J Cell Biol 197: 45-57.

Dieterich DC, Hodas JJ, Gouzer G, Shadrin IY, Ngo JT, Triller A, Tirrell DA, Schuman EM. 2010. In situ visualization and dynamics of newly synthesized proteins in rat hippocampal neurons. Nat Neurosci 13: 897-905.

Gallagher SS, Sable JE, Sheetz MP, Cornish VW. 2009. An in vivo covalent TMP-tag based on proximity-induced reactivity. ACS Chem Biol 4: 547-556.

Gautier A, Juillerat A, Heinis C, Correa IR Jr, Kindermann M, Beaufils F, Johnsson K. 2008. An engineered protein tag for multiprotein labeling in living cells. Chem Biol 15: 128-136.

Graber TE, Hebert-Seropian S, Khoutorsky A, David A, Yewdell JW, Lacaille JC, Sossin WS. 2013. Reactivation of stalled polyribosomes in synaptic plasticity. Proc Natl Acad Sci 110: 16205-16210.

Griffin BA, Adams SR, Tsien RY. 1998. Specific covalent labeling of recombinant protein molecules inside live cells. Science 281: 269-272.

Grimm JB, English BP, Chen J, Slaughter JP, Zhang Z, Revyakin A, Patel R, Macklin JJ, Normanno D, Singer RH, et al. 2015. A general method to improve fluorophores for live-cell and single-molecule microscopy. Nat Methods 12: $244-250$.

Grimm JB, English BP, Choi H, Muthusamy AK, Mehl BP, Dong P, Brown TA, Lippincott-Schwartz J, Liu Z, Lionnet T, et al. 2016. Bright photoactivatable fluorophores for single-molecule imaging. Nat Methods 13: 985-988.

Halstead JM, Lionnet T, Wilbertz JH, Wippich F, Ephrussi A, Singer RH, Chao JA. 2015. Translation. An RNA biosensor for imaging the first round of translation from single cells to living animals. Science 347: 1367-1671.

Hengst U, Deglincerti A, Kim HJ, Jeon NL, Jaffrey SR. 2009. Axonal elongation triggered by stimulus-induced local translation of a polarity complex protein. Nat Cell Biol 11: 1024-1030.

Holt CE, Schuman EM. 2013. The central dogma decentralized: New perspectives on RNA function and local translation in neurons. Neuron 80: 648-657.

Ifrim MF, Williams KR, Bassell GJ. 2015. Single-molecule imaging of PSD-95 mRNA translation in dendrites and its dysregulation in a mouse model of fragile $\mathrm{X}$ syndrome. $J$ Neurosci 35: 7116-7130.

* Ingolia NT, Hussmann JA, Weissman JS. 2018. Ribosome profiling: Global views of translation. Cold Spring Harb Perspect Biol doi: 10.1101/cshperspect.a032698.

* Ivanov P, Kedersha N, Anderson P. 2018. Stress granules and processing bodies in translational control. Cold Spring Harb Perspect Biol doi: 10.1101/cshperspect.a032813.

* Karousis ED, Mühlemann O. 2018. Nonsense-mediated mRNA decay begins where translation ends. Cold Spring Harb Perspect Biol doi: 10.1101/cshperspect. a032862.

Katz ZB, English BP, Lionnet T, Yoon YJ, Monnier N, Ovryn B, Bathe M, Singer RH. 2016. Mapping translation "hotspots" in live cells by tracking single molecules of mRNA and ribosomes. eLife 5: e10415. 
J. Biswas et al.

Kiebler MA, Bassell GJ. 2006. Neuronal RNA granules: Movers and makers. Neuron 51: 685-690.

Kos A, Wanke KA, Gioio A, Martens GJ, Kaplan BB, Aschrafi A. 2016. Monitoring mRNA translation in neuronal processes using fluorescent noncanonical amino acid tagging. J Histochem Cytochem 64: 323-333.

Krichevsky AM, Kosik KS. 2001. Neuronal RNA granules: A link between RNA localization and stimulation-dependent translation. Neuron 32: 683-696.

Lecuyer E, Yoshida H, Parthasarathy N, Alm C, Babak T, Cerovina T, Hughes TR, Tomancak P, Krause HM. 2007. Global analysis of mRNA localization reveals a prominent role in organizing cellular architecture and function. Cell 131: 174-187.

Lin MZ, Glenn JS, Tsien RY. 2008. A drug-controllable tag for visualizing newly synthesized proteins in cells and whole animals. Proc Natl Acad Sci 105: 7744-7749.

Liu J, Xu Y, Stoleru D, Salic A. 2012. Imaging protein synthesis in cells and tissues with an alkyne analog of puromycin. Proc Natl Acad Sci 109: 413-418.

Los GV, Encell LP, McDougall MG, Hartzell DD, Karassina N, Zimprich C, Wood MG, Learish R, Ohana RF, Urh M, et al. 2008. HaloTag: A novel protein labeling technology for cell imaging and protein analysis. ACS Chem Biol 3: 373-382.

Moor AE, Golan M, Massasa EE, Lemze D, Weizman T, Shenhav R, Baydatch S, Mizrahi O, Winkler R, Golani O, et al. 2017. Global mRNA polarization regulates translation efficiency in the intestinal epithelium. Science 357: 1299-1303.

Morisaki T, Lyon K, DeLuca KF, DeLuca JG, English BP, Zhang Z, Lavis LD, Grimm JB, Viswanathan S, Looger LL, et al. 2016. Real-time quantification of single RNA translation dynamics in living cells. Science 352: 14251429.

Na Y, Park S, Lee C, Kim DK, Park JM, Sockanathan S, Huganir RL, Worley PF. 2016. Real-time imaging reveals properties of glutamate-induced Arc/Arg 3.1 translation in neuronal dendrites. Neuron 91: 561-573.

Pichon X, Bastide A, Safieddine A, Chouaib R, Samacoits A, Basyuk E, Peter M, Mueller F, Bertrand E. 2016. Visualization of single endogenous polysomes reveals the dynamics of translation in live human cells. J Cell Biol 214: 769-781.

Rodriguez AJ, Shenoy SM, Singer RH, Condeelis J. 2006. Visualization of mRNA translation in living cells. J Cell Biol 175: 67-76.

Rufener SC, Mühlemann O. 2013. eIF4E-bound mRNPs are substrates for nonsense-mediated mRNA decay in mammalian cells. Nat Struct Mol Biol 20: 710-717.

Schmidt EK, Clavarino G, Ceppi M, Pierre P. 2009. SUnSET, a nonradioactive method to monitor protein synthesis. Nat Methods 6: 275-277.

Schwanhausser B, Busse D, Li N, Dittmar G, Schuchhardt J, Wolf J, Chen W, Selbach M. 2011. Global quantification of mammalian gene expression control. Nature 473: 337 342.

Starck SR, Green HM, Alberola-Ila J, Roberts RW. 2004. A general approach to detect protein expression in vivo using fluorescent puromycin conjugates. Chem Biol 11: 999-1008.
Tanenbaum ME, Gilbert LA, Qi LS, Weissman JS, Vale RD. 2014. A protein-tagging system for signal amplification in gene expression and fluorescence imaging. Cell 159: 635646.

Tatavarty V, Ifrim MF, Levin M, Korza G, Barbarese E, Yu J, Carson JH. 2012. Single-molecule imaging of translational output from individual RNA granules in neurons. $\mathrm{Mol}$ Biol Cell 23: 918-929.

tom Dieck S, Kochen L, Hanus C, Heumuller M, Bartnik I, Nassim-Assir B, Merk K, Mosler T, Garg S, Bunse S, et al. 2015. Direct visualization of newly synthesized target proteins in situ. Nat Methods 12: 411-414.

Trcek T, Larson DR, Moldon A, Query CC, Singer RH. 2011. Single-molecule mRNA decay measurements reveal promoter-regulated mRNA stability in yeast. Cell 147: 14841497.

Tutucci E, Vera M, Biswas J, Garcia J, Parker R, Singer RH. 2017. An improved MS2 system for accurate reporting of the mRNA life cycle. Nat Methods 15: 81-89.

Tutucci E, Livingston NM, Singer RH, Wu B. 2018. Imaging mRNA in vivo, from birth to death. Annu Rev Biophys doi: 10.1146/annurev-biophys-070317-033037.

Viswanathan S, Williams ME, Bloss EB, Stasevich TJ, Speer CM, Nern A, Pfeiffer BD, Hooks BM, Li WP, English BP, et al. 2015. High-performance probes for light and electron microscopy. Nat Methods 12: 568-576.

Wang DO, Kim SM, Zhao Y, Hwang H, Miura SK, Sossin WS, Martin KC. 2009. Synapse- and stimulus-specific local translation during long-term neuronal plasticity. Science 324: 1536-1540.

Wang C, Han B, Zhou R, Zhuang X. 2016. Real-time imaging of translation on single mRNA transcripts in live cells. Cell 165: 990-1001.

Wei L, Yu Y, Shen Y, Wang MC, Min W. 2013. Vibrational imaging of newly synthesized proteins in live cells by stimulated Raman scattering microscopy. Proc Natl Acad Sci 110: 11226-11231.

Wei L, Hu F, Shen Y, Chen Z, Yu Y, Lin CC, Wang MC, Min W. 2014. Live-cell imaging of alkyne-tagged small biomolecules by stimulated Raman scattering. Nat Methods 11: $410-412$.

Wong HH, Lin JQ, Strohl F, Roque CG, Cioni JM, Cagnetta R, Turner-Bridger B, Laine RF, Harris WA, Kaminski CF, et al. 2017. RNA docking and local translation regulate site-specific axon remodeling in vivo. Neuron 95: 852868.e858.

Wu B, Chen Y, Muller JD. 2010. Heterospecies partition analysis reveals binding curve and stoichiometry of protein interactions in living cells. Proc Natl Acad Sci 107: 4117-4122.

Wu B, Piatkevich KD, Lionnet T, Singer RH, Verkhusha VV. 2011. Modern fluorescent proteins and imaging technologies to study gene expression, nuclear localization, and dynamics. Curr Opin Cell Biol 23: 310-317.

Wu B, Buxbaum AR, Katz ZB, Yoon YJ, Singer RH. 2015. Quantifying protein-mRNA interactions in single live cells. Cell 162: 211-220.

Wu B, Eliscovich C, Yoon YJ, Singer RH. 2016. Translation dynamics of single mRNAs in live cells and neurons. Science 352: 1430-1435. 
Imaging Translation in Single Cells

Yan X, Hoek TA, Vale RD, Tanenbaum ME. 2016. Dynamics of translation of single mRNA molecules in vivo. Cell 165: 976-989.

Yoon YJ, Wu B, Buxbaum AR, Das S, Tsai A, English BP, Grimm JB, Lavis LD, Singer RH. 2016. Glutamate-induced RNA localization and translation in neurons. Proc Natl Acad Sci 113: E6877-E6886.

Younts TJ, Monday HR, Dudok B, Klein ME, Jordan BA, Katona I, Castillo PE. 2016. Presynaptic protein synthesis is required for long-term plasticity of GABA release. Neuron 92: 479-492.

Yu J, Xiao J, Ren X, Lao K, Xie XS. 2006. Probing gene expression in live cells, one protein molecule at a time. Science 311: 1600-1603.
Zappulo A, van den Bruck D, Ciolli Mattioli C, Franke V, Imami K, McShane E, Moreno-Estelles M, Calviello L, Filipchyk A, Peguero-Sanchez E, et al. 2017. RNA localization is a key determinant of neurite-enriched proteome. Nat Commun 8: 583.

Zhou XX, Lin MZ. 2013. Photoswitchable fluorescent proteins: Ten years of colorful chemistry and exciting applications. Curr Opin Chem Biol 17: 682-690.

Zia RKP, Dong JJ, Schmittmann B. 2011. Modeling translation in protein synthesis with TASEP: A tutorial and recent developments. J Stat Phys 144: 405-428.

Zid BM, O’Shea EK. 2014. Promoter sequences direct cytoplasmic localization and translation of mRNAs during starvation in yeast. Nature 514: 117-121. 


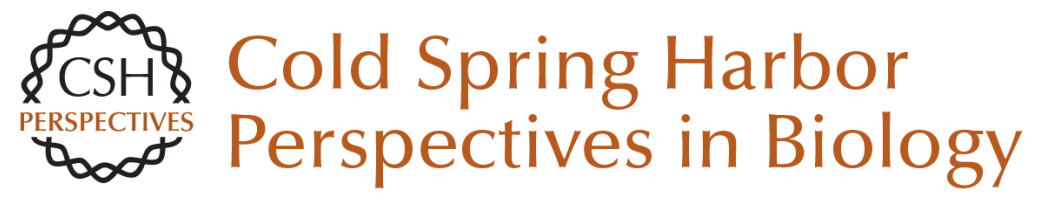

\title{
Fluorescence Imaging Methods to Investigate Translation in Single Cells
}

\author{
Jeetayu Biswas, Yang Liu, Robert H. Singer and Bin Wu
}

Cold Spring Harb Perspect Biol 2019; doi: 10.1101/cshperspect.a032722 originally published online August 6, 2018

\section{Subject Collection Translation Mechanisms and Control}

Protein Synthesis and Translational Control: A

Historical Perspective

Soroush Tahmasebi, Nahum Sonenberg, John

W.B. Hershey, et al.

Translational Control in the Brain in Health and

Disease

Wayne S. Sossin and Mauro Costa-Mattioli

Phosphorylation and Signal Transduction

Pathways in Translational Control Christopher G. Proud

Translational Control during Developmental

Transitions

Felipe Karam Teixeira and Ruth Lehmann

Stress Granules and Processing Bodies in

Translational Control

Pavel Ivanov, Nancy Kedersha and Paul Anderson

Fluorescence Imaging Methods to Investigate

Translation in Single Cells Jeetayu Biswas, Yang Liu, Robert H. Singer, et al.

Translational Control in Virus-Infected Cells Noam Stern-Ginossar, Sunnie R. Thompson, Michael B. Mathews, et al.

Nonsense-Mediated mRNA Decay Begins Where Translation Ends

Evangelos D. Karousis and Oliver Mühlemann
Principles of Translational Control John W.B. Hershey, Nahum Sonenberg and Michael B. Mathews

The Epitranscriptome in Translation Regulation Eyal Peer, Sharon Moshitch-Moshkovitz, Gideon Rechavi, et al.

Translational Control in Cancer Nathaniel Robichaud, Nahum Sonenberg, Davide Ruggero, et al.

Roles of Long Noncoding RNAs and Circular

RNAs in Translation Marina Chekulaeva and Nikolaus Rajewsky

Ribosome Profiling: Global Views of Translation Nicholas T. Ingolia, Jeffrey A. Hussmann and Jonathan S. Weissman

Noncanonical Translation Initiation in Eukaryotes Thaddaeus Kwan and Sunnie R. Thompson

Mechanistic Insights into MicroRNA-Mediated Gene Silencing Thomas F. Duchaine and Marc R. Fabian

Toward a Kinetic Understanding of Eukaryotic Translation Masaaki Sokabe and Christopher S. Fraser

For additional articles in this collection, see http://cshperspectives.cshlp.org/cgi/collection/

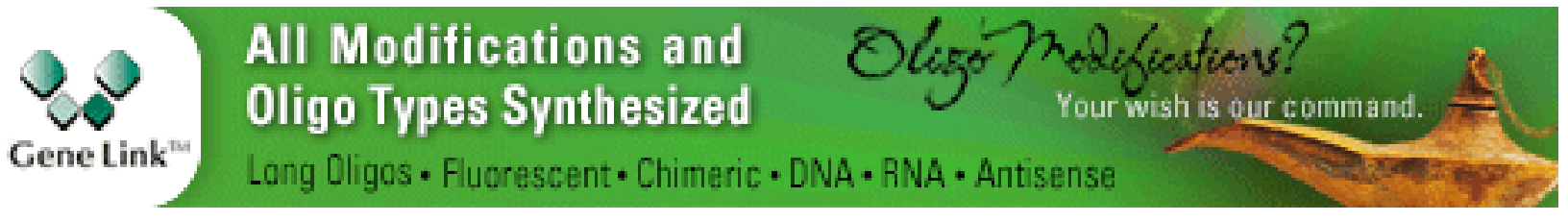


For additional articles in this collection, see http://cshperspectives.cshlp.org/cgi/collection/

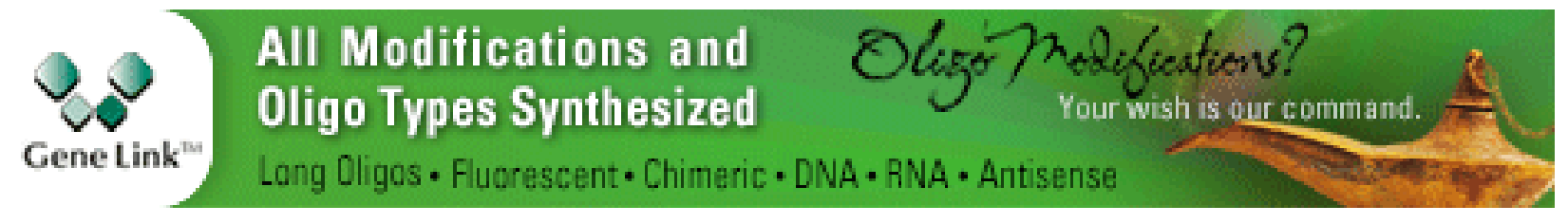

Copyright @ 2019 Cold Spring Harbor Laboratory Press; all rights reserved 